\title{
Advanced Planetary Protection Technologies for the Proposed Future Mission Set
}

\author{
J Andy Spry ${ }^{1}$ \\ JPL/Caltech, Pasadena, CA, 91109, \\ Catharine A Conley ${ }^{2}$ \\ NASA Headquarters, Washington, DC,20546 \\ and \\ Margaret S Race ${ }^{3}$ \\ SETI Institute,Mountain View, CA,94043
}

\begin{abstract}
The discipline of protecting solar system objects from harmful contamination resulting from the activities of interplanetary spacecraft, and of similarly protecting the Earth from uncontrolled release of a putative extra-terrestrial organism from returned extra-terrestrial samples, is called planetary protection. Planetary protection requirements for robotic exploration of Mars have been refined over time as more became understood about the nature of the Martian environment as a potential habitat. Likewise, increased understanding of the limits of life on Earth continues to inform planetary protection policy. In planning for future human exploration of Mars, it is recognized that planetary protection controls for human missions will often be supportive of other important mission needs, such as maximizing closed-loop and recycling capabilities to minimize mass required, minimizing exposure of humans to planetary materials for multiple health reasons, and minimizing contamination of planetary samples and environments during exploration and science activities. In this paper, we outline the progress and plans of a current IAA Study Group that is engaging robotic and human mission developers and scientists in exploring detailed technical, engineering and operational approaches by which planetary protection objectives can be accomplished for human missions. This will be considered through the prism of the planetary protection aspects of the NASA Mars Destination Operations Team, and assessment of current technological capabilities and knowledge gaps.
\end{abstract}

\section{Introduction}

$\mathrm{F}_{\mathrm{e}}^{\mathrm{o}}$ OR nearly five decades, the United Nations (UN) Outer Space Treaty has provided the framework for guiding exploration and activities in outer space. For all this time, missions to diverse solar system bodies have been subject to planetary protection (PP) policies established by the Committee on Space Research (COSPAR) of the International Council for Science. These COSPAR PP policies stipulate that space exploration on the Moon and other celestial bodies shall be done in ways that avoids their harmful contamination (with terrestrial biota).

Current mission plans by NASA and the international space community encompass a variety of mission types that include one way and round trip robotic missions to diverse solar system bodies, as well as anticipated long duration human missions to Mars. Each of these mission types is differentially impacted by PP controls. The focus of PP controls include the avoidance of both forward contamination - the transport of microbial hitchhikers on spacecraft and equipment sent to places like Mars, and back contamination - the return of uncontained extraterrestrial materials (or equipment contaminated with such) to Earth that could harm Earth's biota or ecosystems. In this paper, the principal mission concepts under discussion are those described under the NASA Evolvable Mars Campaign (NASA, 2014). Although this is therefore a US perspective,

\footnotetext{
${ }^{1}$ Planetary Protection Engineer, Spacecraft Mechanical Engineering, JPL (125-224) 4800 Oak Grove Drive, Pasadena CA91109

${ }^{2}$ Planetary Protection Officer, NASA Headquarters (3X63) Washington DC 20546-0001.

${ }^{3}$ Planetary Protection Consultant, SETI Institute, 189 Bernardo Ave, Suite 100, Mountain View, CA 94043.
} 
all space-faring nations considering similar missions are bound by the same treaty framework. It should be noted that there are no planetary protection controls applicable in low Earth orbit, for either robotic or human missions.

For decades, robotic missions - both one way and round trip - to planets, moons, and other objects have been undertaken with appropriate levels of PP controls (e.g. NASA, 2011) aimed at avoiding the harmful contamination of particular celestial bodies and ensuring the integrity of science activities associated missions. In contrast, the only time that planetary protection provisions have been applied to human missions was during the Apollo program, the first and only time that humans have landed on another celestial body. In the decades since the end of the Apollo program, significant advances have occurred in our understanding of both microbiology and potentially habitable planetary environments. Thus, when future human missions venture beyond ISS and Earth orbit, they will need to address a cadre of new science, technology and legal considerations related to planetary protection, particularly for missions to Mars or other potentially habitable bodies.

The intent of COSPAR PP policy is the same whether a mission is conducted robotically or with human explorers. Although the specific implementation requirements may differ, the overall goals are to avoid harmful forward and back contamination during all mission phases. The current PP policy for human missions is based on four main principles and eight implementation guidelines that apply to the entire mission and its varied activities. Although safeguarding the crew and avoiding forward contamination of Mars are clearly important, the current Principles and Guidelines indicate that safeguarding the Earth from potential back contamination is the highest planetary protection priority for future human Mars missions (COSPAR, 2011). While these guidelines provide a clear conceptual approach for future mission planning, detailed engineering constraints and processes for implementing planetary protection on human missions are yet to be developed and will undoubtedly involve significant planning and design challenges in all mission phases. Future human exploration will clearly depend on the design of new systems and technologies to support astronauts and scientific exploration in safe, effective and productive ways throughout the end-to-end mission, while also meeting planetary protection requirements. It is noteworthy that planetary protection provisions are not merely recommendations or suggestions, but rather mandatory planning elements based on international treaty that ideally need to be considered in all human mission systems and subsystem development activities from the start. They are cross cutting in nature, contribute to the generation of requirements, have feed-forward implications, and represent significant time and funding resource considerations for future mission designs.

\section{Anticipated Impacts of Planetary Protectionn}

\section{A. Microbial Diversity and Distribution}

Based on an extensive review of current and past literature (Carr et al., 1999; NRC, 2002a, 2002b; Race \& Rummel, 2000, 2003; Race, et al., 2001a, 2001b, 2002; Bruch et al., 2001; Criswell et al., 2005; MEPAG HEM-SAG, 2008) that describe potential mission architectures for human exploration beyond LEO and for returning Mars samples, there is good agreement about the many technical domains that will be impacted by COSPAR PP requirements during future human missions. In many cases, the needs of planetary protection overlap synergistically with other mission needs. For example, among the types of systems, equipment and operations likely to be impacted by PP concerns are those associated with managing the unavoidable microbial diversity of the human microbiome that will accompany the crew. There will be a need to develop a baseline inventory (La Duc, et al. 2014) of human associated microbes and understand their distribution, abundance, survival and dispersal under space conditions in order to provide information needed to design real-time monitoring systems associated with crew health, life support systems (e.g., air, water, waste recycling, etc.), differential detection of possible Martian microbes (if they exist), and potential effects on engineering systems e.g., to shield against biocorrosion.

\section{B. Hardware Systems}

Planetary protection concerns will also impact the design and operation of the entire spacecraft hardware system, its subsystems, as well as the human interface. For example, applied research on planetary protection will be needed for development of habitat and lab modules; extravehicular activities (EVAs) and equipment; spacesuits and mobile advanced life support (ALS) systems; design and use of sampling devices; cleaning, maintenance and reuse of instruments; in situ resources utilization (ISRU) technologies; subsurface drilling equipment and operations; sample containment handling and transfer; quarantine facilities for individuals and entire crew; decontamination and sterilization methods and technologies; biodiagnostics and crew medical treatment systems; biocontainment and handling facilities in labs. Issues include: food and biological waste handling; closed loop and recycling capabilities; waste recycling and reuse; ingress/egress systems and operation; responses to off-nominal and contamination events, including performance in venting, leakage, dispersal, and shutdown scenarios. 


\section{Related to Science}

As well as the design and use of sampling devices, and the cleaning, maintenance and reuse of instruments used in the science endeavor, planetary protection issues will also impact the design and execution of sample acquisition and analysis. Related also to the issue of astronaut exposure and the concern of back contamination, the proximity of the sampling activity to potential martian 'special regions' (Beaty et al., 2006; Kminek et al., 2010) needs to be considered. Of course, the highest science value sites (those relevant to the origin of life in the solar system) are also those with the highest level of planetary protection concern. To access those high science value sites, the hardware systems need to be appropriate to the sciencedriven sampling and analysis activity.

\section{Related to Back Contamination}

It is the established consensus position that safeguarding the Earth from potential back contamination is the highest planetary protection priority in Mars exploration. For robotic exploration, one methodology is to break the chain of contact with Mars, so that the probability of introduction of an uncontained unsterilized martian particle into the Earth-Moon system is at an acceptably low level. Clearly this would not be achievable for a returning human mission in the paradigm where prior exposure of the astronauts to martian material is considered inevitable. For a returning human mission therefore, mission designers would need to take into account containment technologies (e.g. for Mars samples); decontamination/sterilization approaches (e.g. for returning hardware); and a quarantine/certification process for the astronauts. Technologies for all of these will need to be developed and tested.

\section{Anticipated potential future activities and other information sources}

Recognizing the importance of planetary protection as one of several cross-cutting priorities for future human missions, the International Academy of Astronautics (IAA) initiated a special study - Expanding Options for Implementing Planetary Protection During Human Space Exploration and Robotic Precursor Missions. The study aims at identifying and recommending various ways to implement the existing COSPAR planetary protection policy in the context of international collaborative human missions. The need for the study traces to previous IAA reports (e.g., Hoffman et al. 2011) as well as numerous other international studies and reports on planetary protection and human missions (e.g., Race et al. 2008; Hogan et $\mathrm{al}$, 2007). While there is general agreement about the list of technical domains impacted by the COSPAR PP requirements, specific approaches to address the concerns remain quite general at this time. Ultimately, this ongoing IAA study seeks aims to provide a comprehensive overview of technical and operationally constrained mission design information to help engineers and planners understand more fully how PP requirements for human missions can be practically implemented in ways that overlap with other critical mission needs across various mission domains -- including PP policy, science return, development and operational costs, astronaut safety and health, programmatic risk, and safeguarding Earth upon return.

Over the last couple of years, PP discipline experts have been contributors to ongoing human exploration mission concept planning activities. In particular, detailed discussions on many of the technical domains impacted by PP considerations are available in published workshop reports and articles (e.g. Drake, 2009). The most current discussions of PP concerns are included in the recent amended DRA 5.0 Addendum 2 report, (Drake \& Watts, 2014), that highlights key strategic knowledge gaps, technology developments needed and model systems that can be utilized to perform the needed development work.

Additionally, potential future lunar or asteroid precursor missions would, based on current concepts, offer an opportunity to rehearse operational scenarios under which a future Mars human exploration mission would be performed, and to address some of the quantitative contamination monitoring aspects concerning collection of a pristine sample by a spacecraft system dominated by systems dedicated to the support of its crew, as opposed to a more spartan (and easier to clean) robotic sample acquisition mission.

The Destination Operations (sub)Team of the NASA Human Architecture Team (DOT-HAT) has developed (among other products) a reasonably comprehensive Functional Decomposition of each of the functions and activities of a human Mars exploration mission, based on the DRA5.0 architecture. This is currently the reference resource for the PP input into an ongoing NASA Space Technology Mission Directorate (STMD) roadmapping activity, ensuring that PP technology needs are duly considered in the creation of the future technology development plan. 


\section{Biohazards and Risks for Human ExplorationMissions}

At this time, quantitative PP guidelines for use by mission planners and designers do not exist. This is because planetary protection requirements are likely to evolve in the coming years, together with the mission concepts, and in response to many factors (e.g., rapid changes in scientific information about Mars and other bodies, advances in technology, increased understanding of terrestrial biology, improvements in methodologies for assaying cleanliness and reducing bioburden, revisions in PP policies, etc.). Thus, a conceptual approach will be useful in guiding early discussions about EVAs, ALS, environmental monitoring and controls (EMC) and other aspects of human missions. In developing a conceptual approach that simultaneously addresses planetary protection concerns, considers science needs, and anticipates life support and human requirements, it is necessary first to bound discussions by making some starting assumptions about the nature and anticipated features of human missions.

PP policies for human missions will have three main foci particularly for target bodies like Mars with biological potential: 1) avoiding forward contamination of Mars or interference with scientific exploration from terrestrially-associated microbial contaminants; 2) protecting astronauts from harmful contamination from possible extraterrestrial life forms; and 3) controlling back contamination from the spacecraft, astronauts and materials that are returned to Earth. Other key concepts and assumptions that have guided deliberations about human missions included:

- Like robotic missions, human missions will need to take a conservative approach and assume that martian life exists until data suggests otherwise at a to be determined level of confidence.

- No habitat or EVA system will be fully closed. Therefore missions carrying humans to Mars or other bodies will contaminate the planet.

- The increased capabilities that human explorers can contribute to astrobiological exploration only exist if humanassociated contamination is controlled and understood. It is therefore critical that attempts be made to seek evidence of putative past and/or present extraterrestrial life, especially on Mars, well before these missions occur and to identify, characterize, minimize, and control contamination sources and pathways that would threaten such evidence.

- Safeguarding the Earth from potential back contamination is the highest planetary protection priority in Mars exploration.

- Crew and hardware on Mars will inevitably be exposed to martian materials. To the maximum extent practicable, these exposures should occur under conditions that are controlled and understood.

- To manage mission costs and the potential for back contamination, it is desirable (perhaps essential in many architectures) to leave wastes and other contaminated materials on Mars upon mission completion. The mission would need to deploy mitigation techniques including physical control over release (e.g., containment), possibly in conjunction with active processing (e.g., sterilization) and/or passive exposure to martian adverse surface conditions, to protect Mars by destroying terrestrial life and biomarkers.

From a PP perspective, the primary biohazards of concern during long duration missions center first on microbial, replicating life - whether terrestrial or extraterrestrial in origin, as well as other sources, such as organic contaminants and biomarkers. While the discussions usually focus on normal operations, it is also recognized that off nominal events could increase contamination threat by orders of magnitude with corresponding implications for crew health and safety as well as forward and back contamination concerns.

\section{A. Forward Contaminants of Concern}

As summarized in Table I, the forward contaminants of greatest concern are the hitchhiker Earth microbes that may be deposited by astronauts, spacecraft or equipment, and potentially spread by either natural conditions (e.g. dusts, storms) or mission-associated operations and activities on the planet. Unlike robotic spacecraft that are extensively cleaned and subjected to sterilization processing prior to launch, human explorers will be accompanied by an assortment of internal and external microbes, making human associated microbial burden unavoidable. Thus, while forward contamination cannot be eliminated, it must be completely understood and mitigated through design and operations aimed at controlling release of contaminants at the planetary body.

Special attention will be needed to control the release and spread of microbial contaminants associated with technologies and systems for food and waste handling, EVA's and suits, habitats and lab modules, ingress/egress, waste disposal, gas venting, and various ISRU operations. 
Awareness and control of organic contaminants and biomarkers will also be important concerns for forward contamination because of the potential to interfere with later science and life detection analyses. Because astronauts will work with varied types of robotic hardware during the mission, some of the planetary protection controls deployed during such a mission would likely be reflective of the stringent microbial bioburden reduction and cleanliness requirements currently imposed on robotic missions, and leverage current knowledge in their implementation. In addition to developing effective designs and operations, the ability to repair, maintain, decontaminate, and replace equipment and hardware will be important for reducing forward contamination risks during human exploration.

It will be impossible to have complete knowledge about the entire planet prior to the first human mission, making it important to develop an appropriate access management classification system that minimize the prospects for cross contamination through all mission phases. Precursor robotically collected data will be needed to characterize particular areas and verify whether they are devoid of detectable signatures of martian life and therefore presumed safe for humans. At a minimum, landing areas must be verified in advance as zones of minimal biological risk that are 'safe' for human operations and represent minimal concern for forward contamination (for a complete discussion of possible classification schemes see Criswell, 2005). Because of the increasing interest in the potential for subsurface liquid water on Mars, controls will be needed to minimize forward contamination during sampling and exploration below the planetary surface.

Finally, the very systems that are used to monitor and control the environments in which astronauts will live and operate, could themselves be sources of potential forward contamination (e.g., filters, organic materials used in test systems, technologies used for decontamination etc). Not only will EMC systems be tasked with monitoring, detecting and maintaining safe and healthful environmental conditions for the crew, they must do so in a way that minimizes and controls forward contamination (either microbial or biomarkers). A system that simultaneously scans for evidence of possible martian contaminants and life, both inside and outside, in close to real time, would be extremely desirable.

Table I Biohazards and Risks Associated with Forward Contamination

\begin{tabular}{|c|c|c|}
\hline Hazard & Risk & Solution \\
\hline \multirow[t]{4}{*}{ Earth Microbes } & $\begin{array}{l}\text { Humans as microbial ‘carriers' - } \\
\text { assumed unavoidable }\end{array}$ & $\begin{array}{l}\text { Control contact with Mars; Mitigate by design and } \\
\text { operations }\end{array}$ \\
\hline & $\begin{array}{l}\text { Spacecraft and equipment deliver microbial } \\
\text { contaminants }\end{array}$ & $\begin{array}{l}\text { Clean/Bioburden reduction } \\
\text { Cleanroom assembly } \\
\text { Repair/Maintain/Decontaminate } \\
\text { Need PP "figures of merit" for human missions }\end{array}$ \\
\hline & $\begin{array}{l}\text { Sampling equipment and operations; Special } \\
\text { concern for subsurfaces }\end{array}$ & $\begin{array}{l}\text { Strict cleanliness like robotic missions \& equipment; } \\
\text { Need protocols for repair/maintainance/decontamination }\end{array}$ \\
\hline & $\begin{array}{l}\text { Local vs. widespread contamination, } \\
\text { different risks and standards by location; } \\
\text { Strategy on risks to be informed by } \\
\text { precursor/robotic science }\end{array}$ & $\begin{array}{l}\text { Location Classification System: } \\
\text { Landing Area must be a "Zone of Miminmum Biological } \\
\text { Risk" } \\
\text { Address each of: } \\
\text { - Biological/Science Concerns } \\
\text { - Contamination Concerns } \\
\text { - Human Operation Zones } \\
\text { - Timing and Sequencing of Operations }\end{array}$ \\
\hline \multirow[t]{3}{*}{$\begin{array}{l}\text { Other Hazards of } \\
\text { Concern }\end{array}$} & Planetary surface dusts & $\begin{array}{l}\text { Evaluate properties e.g., Dispersal: } \\
\text { Research on dust and microbial survival to address concern } \\
\text { about spread in human habitation areas }\end{array}$ \\
\hline & $\begin{array}{l}\text { Food and biological waste technologies } \\
\text { (microbial hitchhikers and use of microbial } \\
\text { treatment systems); } \\
\text { ISRU; Other systems? }\end{array}$ & $\begin{array}{l}\text { Controls to be determined } \\
\text { Solid \& liquid wastes contained } \\
\text { Need extremophile research } \\
\text { Address threat of environmental disruption? }\end{array}$ \\
\hline & $\begin{array}{l}\text { EVA, Suits, Habitats (escape of microbes- } \\
\text { ingress/egress; leakage; etc) }\end{array}$ & $\begin{array}{l}\text { Design and operations } \\
\text { Need to inventory/understand microbes (on people v. } \\
\text { equipment.) } \\
\text { Filtered venting of all gases }\end{array}$ \\
\hline $\begin{array}{l}\text { Organic } \\
\text { Contaminants }\end{array}$ & $\begin{array}{l}\text { Unavoidable organic materials as } \\
\text { 'biomarkers'; False positives and impact on } \\
\text { science }\end{array}$ & $\begin{array}{l}\text { Need to understand via inventory and research; Design to } \\
\text { minimize impact on science }\end{array}$ \\
\hline For All Biohazards & $\begin{array}{l}\text { Monitoring; Detection; Alarms; Controls: } \\
\text { Critical inside, outside, and in mobile units, } \\
\text { with microbes and organics included in } \\
\text { monitoring methods etc. }\end{array}$ & $\begin{array}{l}\text { Technology; sensors and methods to perform evaluation to } \\
\text { be determined. } \\
\text { Inside and outside of human habitations need to be } \\
\text { included }\end{array}$ \\
\hline
\end{tabular}




\section{B. Protecting Astronauts}

Biohazards and health risks of concern to astronauts will derive from many sources-including those traditionally associated with space missions (physical, toxic, medical health/safety concerns, immune response, radiation, desiccation, etc.) Additional risks will likely arise from other potentially unprecedented hazards-in particular, those associated with the lengthy mission duration, extreme planetary conditions, mental health challenges, and possible martian microbiology. While all these hazards will be important for mission success, only those associated with potential exposure to martian life forms represent areas of planetary protection concern.

Exposure to martian microbes and materials could occur in many ways during the mission (e.g. EVAs or suit leaks; during sampling or scientific analysis; in water, food or air systems, from dusts associated with habitation ingress/egress; etc.) Solutions for minimizing, eliminating or mitigating astronaut exposures to extraterrestrial microbes are similar to those for forward contamination control, such as environmental monitoring and control systems, decontamination procedures, use of classification systems to minimize exposures, using special care and equipment during exploration into unknown or subsurface areas, etc. (Table II). In addition, it will be important to avoid the creation of hybrid microenvironments where microbes from both Earth and the target body could mix, leading to concerns about a possible ' 3 rd ecology' with unknown outcomes for crew members (e.g., in air, water or food systems, in laboratory modules, etc).

Because astronauts could come into contact with extraterrestrial microbes or uncharacterized biohazards at any time during a martian surface mission (including on the return journey), quarantine provisions (details TBD) will be needed in situ to isolate, monitor and treat crew member/s that might have been exposed.

\section{Table II: Chem/Biohazards and Risks to Astronauts During Mission}

\begin{tabular}{|c|c|c|}
\hline Hazard & Risk & Solution \\
\hline $\begin{array}{l}\text { Martian } \\
\text { Biohazards }\end{array}$ & $\begin{array}{l}\text { Physical Health/Safety } \\
\text { (Chemical, Radiation, Desiccation etc.) }\end{array}$ & $\begin{array}{l}\text { To be determined: Modify operations } \\
\text { such as medical monitoring and safeguards for Mars }\end{array}$ \\
\hline \multirow[t]{4}{*}{ Martian Microbes? } & $\begin{array}{l}\text { Astronauts exposed to Martian } \\
\text { Life(?)/Dusts (EVAs, Lab, Food, etc.) }\end{array}$ & $\begin{array}{l}\text { Monitoring and control; Decontamination; } \\
\text { Separation of modules; } \\
\text { Quarantine capabilities }\end{array}$ \\
\hline & $\begin{array}{l}\text { Exposures beyond landing area (the } \\
\text { Zone of Minimal Biological Risk) }\end{array}$ & $\begin{array}{l}\text { Classification System for location based on: } \\
\text { Biological/Science Concerns } \\
\text { Contamination Concerns } \\
\text { Human Operation Zones } \\
\text { Operational Timing And Sequencing }\end{array}$ \\
\hline & $\begin{array}{l}\text { Special concerns for subsurface and } \\
\text { unknown regions during sampling \& } \\
\text { exploration }\end{array}$ & $\begin{array}{l}\text { Human-Robotic pairings; } \\
\text { Strict cleanliness requirements like on robotic } \\
\text { missions; } \\
\text { Sterilization of sampling equipment } \\
\text { Repair/Maintain/Decontaminate }\end{array}$ \\
\hline & $\begin{array}{l}3^{\text {rd }} \text { Ecology Concerns } \\
\text { (Creation Of Earth-Mars hybrid } \\
\text { microenvironments?) }\end{array}$ & $\begin{array}{l}\text { Special attention on waste treatment systems and food } \\
\text { generation; } \\
\text { Monitoring of environments }\end{array}$ \\
\hline
\end{tabular}

\section{Back Contamination Concerns}

The main biohazards and risks of concern associated with back contamination are linked with putative replicating martian microbes. As shown in Table III, the risks and the ways to address them are similar to those used for minimizing forward contamination, but the microbial life is presumed to be of martian origin, with potentially unknown characteristics, at least initially. The key to dealing with the risks will be through avoiding situations, technologies or operations that would cause crew, support systems, hardware or returning spacecraft to be exposed to martian dusts, materials or microbes in ways that cause them to be returned to Earth in an uncontained fashion. While the long duration of the return flight will allow ongoing medical monitoring and impose a quarantine of sorts, there will need to be provisions in mission architecture for the quarantine of crew members on Earth. Other unusual risks that will require special attention include the difficulty of monitoring and detecting possible martian 
microbes of unknown characteristics (a task which overlaps to some degree with science exploration per se) and the behavioral or debilitation risks that could contribute to possible implementation lapses of planetary protection controls or safeguards. It will be important that PP oversight and responsibility be assigned to a designated crewmember as well as integrated into flight and operational plans throughout the mission.

Table III: Biohazards and Risks of Concern Regarding Back Contamination of Earth

\begin{tabular}{|c|c|c|}
\hline Biohazard & Risk & Solution \\
\hline \multirow[t]{9}{*}{$\begin{array}{l}\text { Mars } \\
\text { Replicating Microbes? }\end{array}$} & Impossible to break contact with Mars & $\begin{array}{l}\text { Mission architecture and operations to limit contact } \\
\text { with Mars materials; Leave wastes and extra } \\
\text { materials behind upon return? }\end{array}$ \\
\hline & $\begin{array}{l}\text { Uncertainty about existence or extent of } \\
\text { Martian life }\end{array}$ & $\begin{array}{l}\text { Classification System"Safe" zones; Zones of } \\
\text { Minimal Biological Risk; Emphasis on avoiding } \\
\text { exposure in 'Special Regions' }\end{array}$ \\
\hline & EVA, Suits, Rovers, Habitats etc. & $\begin{array}{l}\text { New technologies designed to minimize possibility } \\
\text { of cross contamination with martian materials; } \\
\text { Technologies for cleaning and decontamination }\end{array}$ \\
\hline & $\begin{array}{l}\text { Transfer of lab. and exploration samples, } \\
\text { particularly from uncleared Zones or subsurface }\end{array}$ & $\begin{array}{l}\text { Strict separation and containment of pristine } \\
\text { materials (Similar to robotic missions?) }\end{array}$ \\
\hline & Potential for $3^{\text {rd }}$ Ecology? & $\begin{array}{l}\text { Treatment and waste handling systems designed to } \\
\text { avoid mixes of viable Earth microbes and Martian } \\
\text { materials }\end{array}$ \\
\hline & $\begin{array}{l}\text { Food production, water treatment, off-nominal } \\
\text { events, and misc. systems, etc. }\end{array}$ & $\begin{array}{l}\text { To be determined: Liquid and solid wastes } \\
\text { contained; Special planning for off nominal events } \\
\text { (e.g.: Fire suppression, depressurization, etc.) }\end{array}$ \\
\hline & What if Crew are exposed? & $\begin{array}{l}\text { Medical monitoring and quarantine as part of } \\
\text { mission architecture on Mars and Earth }\end{array}$ \\
\hline & On-going monitoring, detection \& control & $\begin{array}{l}\text { Need to incorporate PP into monitoring, alarm and } \\
\text { control systems }\end{array}$ \\
\hline & PP Oversight & $\begin{array}{l}\text { Designated crew member for PP; Human Factors } \\
\text { research }\end{array}$ \\
\hline
\end{tabular}

\section{Planetary Protection Approach on Human Exploration Missions}

After considering the anticipated biological risks in combination with science exploration considerations, the following tentative conceptual approach was developed as a way to address planetary protection concerns for future human long duration missions to Mars:

- Human missions to Mars shall not adversely affect or contaminate "special regions" (Beaty, 2006) on Mars. This means that mission cleanliness requirements (for orbiter, lander, rovers, crew, instruments, and tools) shall be determined in such a way as to avoid the inadvertent introduction of Earth organisms or organic molecules into these environments, should they exist. Landing site selection and operational accessibility to scientifically desirable sites that may include "special regions" (including access by ISRU activities to important subsurface ice or water) shall be directly traded against the increased microbial and/or organic cleanliness requirements of human-associated (or robotic) systems supporting the missions.

- Calculations of planetary protection control levels based on this approach (contamination avoidance for special regions) will determine the tolerable levels of contamination allowed for specific aspects of any particular human mission. Specific details of the approach will be developed in the future by working closely with the scientific community to integrate changing knowledge, or new information about the martian environment and conditions, and to evaluate and understand the unavoidable levels of human associated contaminants and their implications, given the risk that potential biomarkers may interfere with scientific investigations and/or the martian environment.

In addition, the sub-groups in the Safe on Mars study (NRC 2002b) identified the following special issues that relate to the integration of PP concerns throughout mission planning and operations:

1. The first human mission to Mars will face significant levels of risk during the various phases of the mission. Planetary protection risks must be among the many risks to be identified and evaluated 
together - then reduced, mitigated, or eliminated when possible to enable mission success. Close and early coordination is essential among the diverse groups of specialists working on the many phases of mission development.

2. General human factors need to be considered along with planetary protection issues for a human mission to Mars. Physical effects that may lead to debilitation and reduced performance in astronauts could also lead to unintended actions and, in turn, to mishaps with potentially serious planetary protection consequences. Mistakes are much more likely when people are tired, ill, and/or overly stressed

Planetary protection is intimately linked with crew health and safety as well as overall mission success; it must be given appropriate priority in every mission phase. A crewmember onboard the mission should be given primary responsibility for the implementation of planetary protection provisions affecting the crew during the mission. In addition, PP information must be emphasized in the development of mission operations plans and medical oversight throughout the mission.

\section{Conclusions}

Although there is an absence of detailed PP policies and requirements for human missions at present, it is possible to outline a conceptual approach and provide preliminary guidelines for planners and designers of e.g., EVA, ALS, and EMC systems. This approach for Mars human exploration missions is based on an understanding of anticipated biohazards as well a growing understanding of martian environmental conditions and life support and habitation needs.

Iteration and refinement of the approach and development of more specific guidelines will likely occur over time in response to information from research and technology development activities, coupled with findings from the planned precursor robotic missions expected in the coming decade and beyond. What is clear however, is that PP requirements for human missions will undoubtedly be very different than those used during the Apollo missions.

\section{Acknowledgments}

This work was carried out in part at the Jet Propulsion Laboratory, California Institute of Technology, under a contract with the National Aeronautics and Space Administration.

\section{References}

Beaty, D.W. et al. (2006) Findings of the Special Regions Science Analysis Group; Astrobiology 6 p677-732

Bruch, C.W., R.B. Setlow, and J.D. Rummel (eds.) (2001) Mars Sample Handling Protocol Workshop Series: Workshop 2a. Sterilization Workshop, Proceedings and Final Report, NASA/CP-2001-210924 (June 2001), NASA, Washington DC

Carr, M., et al., (1999) Mars Sample Handling and Requirements Panel (MSHARP) Final Report, Jet Propulsion Laboratory, Pasadena CA, NASA TM-1999-209145

COSPAR Planetary Protection Policy (2002, as amended 2011) https://194.199.174.76/sites/default/files/pppolicy.pdf (accessed 4/2014)

Criswell, M.E., et al., (2005) Planetary Protection Issues in the Human Exploration of Mars, Final Report May 9, 2005 (workshop held June 2001), NASA, Ames Research Center, Moffett Field CA, NASA/CP - 2005-213461

Drake, B. G. and K. D. Watts, (editors) (2014) Human Exploration of Mars; Design Reference Architecture 5.0 Addendum \#2 NASA/SP-2009-566-ADD2, NASA Johnson Space Center, Houston, Texas

Drake, B. G. (editor) (2009) Human Exploration of Mars; Design Reference Architecture 5.0 NASA/SP-2009-566, NASA Johnson Space Center, Houston, Texas 


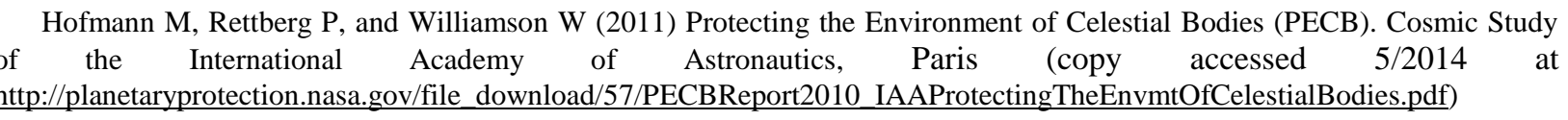

Hogan J.A. et al.(2007) Results Summary of the Life Support and Habitation and PP Workshop, Paper No.2006-01-2007. SAE 2006, Transactions of Journal of Aerospace, March 2007

Kminek, G. et al.(2010) Report of the COSPAR Mars Special Regions Colloquium: Adv Space Res 46 p811-829

La Duc, M. T., Venkateswaran, K., and Conley, C. A.(2014) A Genetic Inventory of Spacecraft and Associated Surfaces; Astrobiology14(1): 15-23

MEPAG HEM-SAG (2008) Planning for the Scientific Exploration of Mars by Humans (unpublished white paper), posted at http://mepag.jpl.nasa.gov/reports/index.html (accessed 4/2014)

NASA, (2011) Planetary Protection Provisions for Extraterrestrial Missions, NPR 8020.12D (see Documents link on NASA Planetary Protection website: www.planetaryprotection.nasa.gov, accessed 4/2014)

NASA, (2014) An Evolvable Mars Campaign (see http://www.nasa.gov/sites/default/files/files/20140429-Crusan-EvolvableMars-Campaign.pdf, accessed 5/2014)

NRC, (2002a) The Quarantine and Certification of Martian Samples. Committee on Planetary and Lunar Exploration (COMPLEX), National Research Council. National Academy Press, Washington DC. (www.nap.edu/books/0309075718/html/index.html, accessed 4/2014)

NRC, (2002b) Safe on Mars: Precursor Measurements Necessary to Support Human Operations on the Martian Surface, National Academy Press, Washington DC, http://books.nap.edu/catalog.php?record_id=10360, accessed 4/2014.

Race, M.S. and J.D. Rummel (eds.), (2000) Mars Sample Handling Protocol Workshop Series. Interim Report of the Workshop Series: Workshop 1 Proceedings and Final Report. NASA/CP-2000-209624, Washington, DC (Oct. 2000)

Race, M. S., K. Nealson, Rummel, J.D. and S. E. Acevedo (eds.) (2001a) Mars Sample Handling Protocol Workshop Series. Interim Report of the Workshop Series: Workshop 3 Proceedings and Final Report. NASA/CP-2001- 211388, Washington, D.C.

Race, M. S., G.T.A. Kovacs, J.D. Rummel, and S. E. Acevedo (eds.), (2001b) Mars Sample Handling Protocol Workshop Series. Interim Report of the Workshop Series: Workshop 2 Proceedings and Final Report, NASA/CP 2001-210923, Washington, D.C.

Race, M.S., D.L.DeVincenzi, J.D.Rummel and S.E.Acevedo (eds.), (2002) Mars Sample Handling Protocol Workshop Series, Workshop 4 Proceedings and Final Report. NASA/CP-2002-21184, Washington, D.C.

Race, M.S. and J.D. Rummel. (2003) Planning for Mars Sample Return: Design and Implementation Considerations for Handling and Testing Returned Samples. ICES Paper No. 2003-ICES-403 (invited), International Conference on Environmental Systems (ICES), Vancouver, BC, July 2003

Race, M.S., G. Kminek and J.D. Rummel (eds) (2008) Planetary protection and humans on Mars: NASA/ESA workshop results, Adv Space Res 42 p1128-1138

Rummel, J.D., M.S. Race, D.L. DeVincenzi, P.J. Schad, P.D. Stabekis, M. Viso and S. E. Acevedo (eds.), (2002) A Draft Test Protocol for Detecting Possible Biohazards in Martian Samples Returned to Earth, NASA/CP-2002-211842, Washington D.C. 